\title{
A Critical Review of Analytical Methods for Determination of Ceftriaxone Sodium
}

\section{Mariana Teixeira da Trindade \& Hérida Regina Nunes Salgado}

To cite this article: Mariana Teixeira da Trindade \& Hérida Regina Nunes Salgado (2018) A Critical Review of Analytical Methods for Determination of Ceftriaxone Sodium, Critical Reviews in Analytical Chemistry, 48:2, 95-101, DOI: $10.1080 / 10408347.2017 .1398063$

To link to this article: https://doi.org/10.1080/10408347.2017.1398063

册Published online: 29 Jan 2018.

Submit your article to this journal $\pi$

Џ Article views: 95

Citing articles: 2 View citing articles $\sqsubset$ 


\title{
A Critical Review of Analytical Methods for Determination of Ceftriaxone Sodium
}

\author{
Mariana Teixeira da Trindade (D) and Hérida Regina Nunes Salgado (D) \\ Department of Pharmaceutics, School of Pharmaceutical Sciences, Universidade Estadual Paulista, Araraquara, SP, Brazil
}

\begin{abstract}
Ceftriaxone sodium is a third-generation semi-synthetic antibiotic belonging to the class of cephalosporins. Is administered only by parenteral route and has the ability to cross the blood-brain barrier. It has bactericidal action; its main activity is related to the Gram-negative bacteria, being also able to act against Gram-negative bacilli resistant to the first- and second-generation cephalosporins. The present study presents a survey of the characteristics, properties and analytical methods used for the determination of ceftriaxone sodium, for the gathering of data searches were carried out in scientific articles in the world literature, as well as in the official compendia. It is necessary to create awareness about the importance of developing effective and reliable analytical methods for quality control and consequently for conducting pharmacokinetic, bioavailability, bioequivalence studies as well as for the therapeutic monitoring of this drug. Most of the methods found use high-performance liquid chromatography, but also methods that use absorption spectroscopy ultraviolet, infrared spectroscopy, spectrofluorimetry and microbiological methods have been presented. A discussion was presented highlighting the need to develop new ecological methods using less toxic solvents, rapid analysis and miniaturization of the samples.
\end{abstract}

\section{KEYWORDS}

Analytical methods; ceftriaxone sodium; quality control; review

\section{Introduction}

Natural antibiotics and their semi-synthetic derivatives comprise the majority of antibiotics in clinical use; the class of $\beta$-lactams constitutes the first class of derivatives of natural products used in the therapeutic treatment of bacterial infections. ${ }^{[1]}$

Since 1970, cephalosporins are among the most potent and widely used anti-infective agentes. ${ }^{[2]}$ They are the second largest class of $\beta$-lactam antibiotics which have a broad spectrum of antibacterial activity, clinical efficacy and excellent safety profile, acting on the enzyme transpeptidase, which is unique in bactéria which confers the action in impediment of synthesis of the wall bacterial. ${ }^{[1]}$ Cephalosporins are classified into generations according to general characteristics of antimicrobial activity. Currently, there are five generations of cephalosporins. They differ in relation to the action spectrum, stability to $\beta$-lactamase, pharmacokinetics, stability and collateral reactions. ${ }^{[2-5]}$

Ceftriaxone sodium is a third-generation semi-synthetic cephalosporin, derived from a fermentation product, for parenteral use, being this group of extreme importance because they are able to overcome the blood-brain barrier, since previous generations do not have this capacity. ${ }^{[1,6,7]}$

It acts on Gram-positive and Gram-negative bacteria, the activity against Gram-positive is noticeably smaller in relation to the first-generation cephalosporins. Its major activity is related to Gram-negative bacteria, and it is also capable of acting against Gram-negative bacilli resistant to the first- and second-generation cephalosporins. ${ }^{[2,7,8,9]}$
Ceftriaxone is presented in the form of single-ingredient preparations in the USA, United Kingdom, Japan and Canada as Rocephin $^{\mathrm{TM}}$; in Brazil as Rocefin ${ }^{\mathrm{TM}}$, Ceftriax $^{\mathrm{TM}}$, Celltriaxon ${ }^{\mathrm{TM}}$, Keftron $^{\mathrm{TM}}$, Triaxin ${ }^{\mathrm{TM}}$, Amplospec $^{\mathrm{TM}}$, Ceftriona $^{\mathrm{TM}}$, Triaxton $^{\mathrm{TM}}$; in China as Ansailong ${ }^{\mathrm{TM}}$, Cefin $^{\mathrm{TM}}$, Dezhi ${ }^{\mathrm{TM}}$, Likang Kesong $^{\mathrm{TM}}$, Livzonphin ${ }^{\mathrm{TM}}$, Locekin ${ }^{\mathrm{TM}}$, Oframax ${ }^{\mathrm{TM}}$, Rocephin ${ }^{\mathrm{TM}}$, Xianqin ${ }^{\mathrm{TM}}$; in Germany as Cefotrix ${ }^{\mathrm{TM}}$, Rocephin ${ }^{\mathrm{TM}}$; in Italy as Axobat $^{\mathrm{TM}}$, Bixon $^{\mathrm{TM}}$, Cefrag $^{\mathrm{TM}}$, Davixon ${ }^{\mathrm{TM}}$, Daytrix ${ }^{\mathrm{TM}}$, Deixim $^{\mathrm{TM}}$, Diaxone ${ }^{\mathrm{TM}}$, Eftry $^{\mathrm{TM}}$, Eraxitron ${ }^{\mathrm{TM}}$, Fidato ${ }^{\mathrm{TM}}$, Frieng ${ }^{\mathrm{TM}}$, Kocefan $^{\mathrm{TM}}$, Monoxar ${ }^{\mathrm{TM}}$, Nilson ${ }^{\mathrm{TM}}$, Panatrix ${ }^{\mathrm{TM}}$, Pantoxon ${ }^{\mathrm{TM}}$,

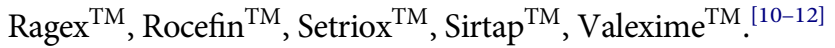

It is indicated for cases of septicemia, meningitis, disseminated Lyme borreliosis (early and late stages of the disease) (Lyme disease), intra-abdominal infections (peritonitis, gastrointestinal and biliary tract infections), bone, joint, soft tissue, skin and wound infections, infections in immunocompromised patients, kidney and urinary tract infections; infections of the respiratory tract, particularly pneumonia andotolaryngological infections, genital infections, including gonorrhea, perioperative prophylaxis of infections. ${ }^{[10,11,13]}$

The development of effective and reliable analytical methods is extremely important for quality control of marketed drugs, being this a multidisciplinary task. The need to demonstrate such efficacy and trust is increasingly recognized and demanded. Therefore, the validation process of the analysis is fundamental to guarantee the analytical quality, providing reliability in the obtained results. ${ }^{[3,4,14,15]}$

CONTACT Prof Hérida Regina Nunes Salgado salgadoh@fcfar.unesp.br $\Theta$ School of Pharmaceutical Sciences, Universidade Estadual Paulista, Araraquara, SP, Brazil. 


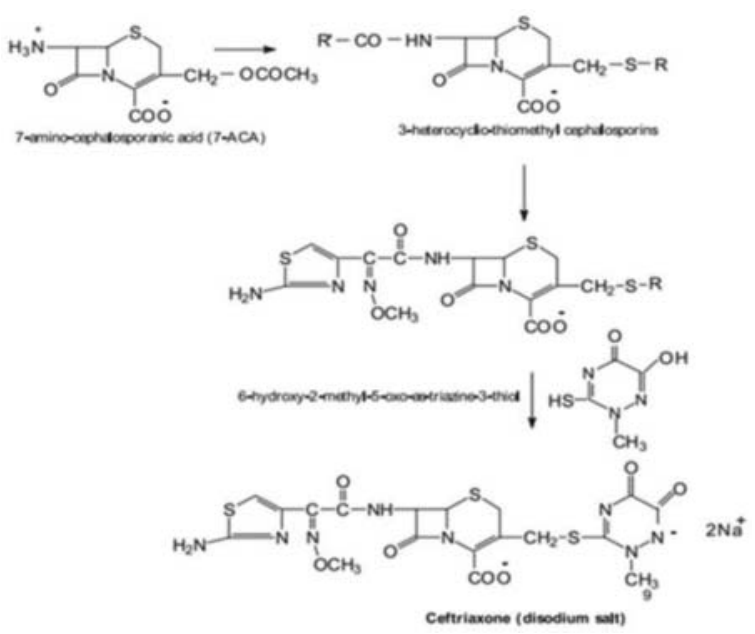

Figure 1. Synthesis of ceftriaxone sodium.

Due to the importance of this information, the present study presents a review of the characteristics of ceftriaxone sodium, its properties and the analytical methods used for its identification and quantification.

\section{Ceftriaxone sodium}

\section{Structural modification}

Ceftriaxone sodium was synthesized through the precursor 7-amino-cephalosporanic acid (7-ACA). Synthesis occurred by the addition of an acyl molecule at the 7-amino position and the displacement of the acetoxy group by thio-substituent heterocyclic at the 3-methyl position. Then, the substitution of the R-alkyl group was determined by the addition of 6-hydroxy-2-methyl-5-oxythiadiazine-3-thiol, the RO-alkyl group was substituted by 2- (2amino-4-thiazolyl) -2-amino- [(Z) -methoxylamino] acetyl completing the synthesis of ceftriaxone sodium. The model for the synthesis of ceftriaxone sodium is shown in Figure 1. ${ }^{[16]}$

\section{Structural forms}

Ceftriaxone sodium is present in disodium hemieptahydrate form, its molecular structure is observed in Figure 2..$^{17-22]}$

\section{Action mechanism}

Ceftriaxone sodium has a bactericidal action, through the inhibition of cell wall synthesis. It is highly stable in the most $\beta$-lactamases, shows greater activity against Gram-negative bacteria

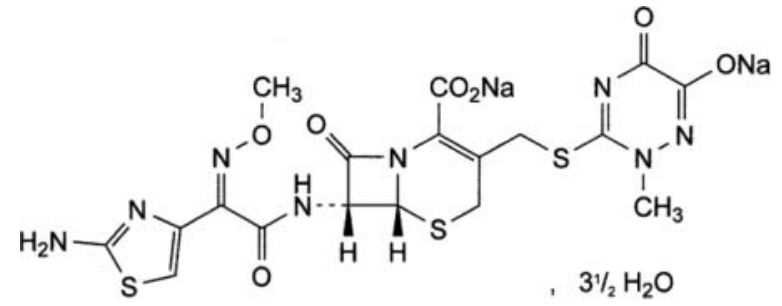

Figure 2. Chemical structure of ceftriaxone disodium hemieptahydrate (CAS 104376-79-6). ${ }^{[17]}$ and is also capable of acting against Gram-negative bacilli resistant to the first- and second-generation cephalosporins, it is effective against the enterobacteria Haemophilus influenzae and Streptococcus pneumoniae, as well as Citrobacter, Serratia marcescens and Providencia. It has synergistic action with the aminoglycosides. ${ }^{[8,23,24]}$

\section{Pharmacokinetics}

Ceftriaxone sodium is administered parenteral route and is not absorbed by the oral route. The daily dose of $1 \mathrm{~g}$ is satisfactory for most serious infections, it is recommended to administer $4 \mathrm{~g}$ once daily in the treatment of meningitis. It has a half-life between 6 and 9 hours and may be prolonged in neonates. This cephalosporin can be injected every 24 hours at a dose of 15$50 \mathrm{mg} / \mathrm{kg} / \mathrm{day}$, it has satisfactory penetration of body fluids and tissues, it reaches sufficient levels in the cerebrospinal fluid to inhibit most pathogens except Pseudomonas, it is more active against pneumococci resistant to penicillin. About 85 to $95 \%$ is bound to plasma proteins. Crosses the placenta and low concentrations have been detected in breast milk; ceftriaxone is excreted unchanged in the urine ( 40 to $65 \%$ ), the remainder is excreted in the biliary tract and there is no need for dose adjustment in renal insufficiency. ${ }^{[10,23,24,25]}$

\section{Physicochemical properties}

Ceftriaxone sodium is a semi-synthetic antibiotic derived from a fermentation product chemically designated asdisodium $(6 R, 7 R)-7-[[(2 Z)-(2$-aminothiazol-4-yl)(methoxyimino)acetyl $]$ amino]-3-[[(2-methyl-6-oxido-5-oxo-2,5-dihydro-1,2,4-triazin-3-yl)sulfanyl]methyl]-8-oxo-5-thia-1-azabicyclo[4.2.0]oct2-ene-2-carboxylate 3.5 hydrate. ${ }^{[17]}$

It has the molecular formula $\mathrm{C}_{18} \mathrm{H}_{16} \mathrm{~N}_{8} \mathrm{Na}_{2} \mathrm{O}_{7} \mathrm{~S}_{3}, 3 \frac{1}{2} \mathrm{H}_{2} \mathrm{O}$, with molecular weight of $661.60 \mathrm{~g} / \mathrm{mol}$ and for the anhydrous form $\left(\mathrm{C}_{18} \mathrm{H}_{18} \mathrm{~N}_{8} \mathrm{O}_{7} \mathrm{~S}_{3}\right) 598.56 \mathrm{~g} / \mathrm{mol}^{[22]}$

Ceftriaxone sodium is in the form of a crystalline powder, almost white or yellowish, slightly hygroscopic, very soluble in water, poorly soluble in methanol, very sparingly soluble in ethanol. It has at least $905 \mu \mathrm{g}$ (potency) and not more than $935 \mu \mathrm{g} / \mathrm{mg}$ calculated on the anhydrous basis. ${ }^{[17-19]}$ It shows melting point $>155^{\circ} \mathrm{C}, \log \mathrm{P}-1.7$ with pka 3.19 (acid) and 4.17 (alkaline). ${ }^{[26]}$

\section{Analytical methods for determining the ceftriaxone sodium}

The analytical methods for ceftriaxone evaluation were researched in the literature through scientific articles, as well as in official compendium (Portuguese Pharmacopoeia, 2005; SP, 2007; JP, 2011; BP, 2013; EP, 2013; USP, 2016). The sites used during a survey were: http://www.sciencedirect.com/, http:// www.scopus.com/ and http://www.ncbi.nlm.nih.gov/pubmed/. Key words were used: ceftriaxone sodium, analytical methods and green chemistry. The research was carried out from July to September 2017.

Quantitation of the ceftriaxone sodium is of the utmost importance for conducting pharmacokinetic studies of bioavailability and bioequivalence therefore for the therapeutic 
Table 1. Chromatographic analytical methods described in the literature for the determination of ceftriaxone sodium.

\begin{tabular}{|c|c|c|c|c|}
\hline Method & Conditions & Detection system & Matrices & Reference \\
\hline HPLC-UV & $\begin{array}{l}\text { Column } \mathrm{C} 18(250 \mathrm{~mm} \times 4.6 \mathrm{~mm} ; 5 \mu \mathrm{m}) \text {. Mobile phase: dissolve } 2 \mathrm{~g} \text { of } \\
\text { tetradecyl ammonium bromide and } 2 \mathrm{~g} \text { of tetraheptyl ammonium } \\
\text { bromide in a mixture of } 440 \mathrm{~mL} \text { of water, } 55 \mathrm{~mL} \text { phosphate buffer } \\
\mathrm{pH} 7,5.0 \mathrm{~mL} \text { citrate buffer pH } 5 \text { and } 500 \mathrm{~mL} \text { of acetonitrile; flow } \\
\text { rate } 1.5 \mathrm{~mL} / \mathrm{min} \text {; injection volume } 20 \mu \mathrm{L} \text {. }\end{array}$ & $254 \mathrm{~nm}$ & Standard & {$[17-22]$} \\
\hline HPLC-UV & $\begin{array}{l}\text { Column hypersil gold C18 }(100 \mathrm{~mm} \times 4.6 \mathrm{~mm} ; 10 \mu \mathrm{m}) \text {; at ambient } \\
\text { temperature. Mobile phase: methanol: } 0.025 \mathrm{M} \text { monopotassium } \\
\text { phosphate adjusted to } \mathrm{pH} 7.5 \text { using triethylamine }(16: 84, \mathrm{v} / \mathrm{v}) \text {; flow } \\
\text { rate } 1 \mathrm{~mL} / \mathrm{min} \text {; injection volume } 10 \mu \mathrm{L} \text {. }\end{array}$ & $254 \mathrm{~nm}$ & $\begin{array}{l}\text { Standard and pharmaceutical } \\
\text { form }\end{array}$ & [29] \\
\hline HPLC-UV & $\begin{array}{l}\text { Column ODS hypersil C18 }(250 \mathrm{~mm} \times 4.6 \mathrm{~mm} ; 5 \mu \mathrm{m}) \text {. Mobile phase: } \\
50 \mathrm{mM} \text { ammonium phosphate buffer and methanol }(90: 10 \% \mathrm{v} / \mathrm{v}) \\
\text { adjusted to } \mathrm{pH} 7.0 \text { with triethylamine; flow rate } 1 \mathrm{~mL} / \mathrm{min} \text {; injection } \\
\text { volume } 20 \mu \mathrm{L} \text {. }\end{array}$ & $230 \mathrm{~nm}$ & Parenteral formulation & [36] \\
\hline HPLC-UV & $\begin{array}{l}\text { Column waters } \times \text { Terra RP-18 }(250 \mathrm{~mm} \times 4.6 \mathrm{~mm} ; 5 \mu \mathrm{m}) \text {. Mobile } \\
\text { phase: } 0.1 \mathrm{M} \text { triethylammonium acetate and acetonitrile }(60: 40 \mathrm{v} / \\
\text { v); flow rate } 1 \mathrm{~mL} / \mathrm{min} \text {; injection volume } 20 \mu \mathrm{L} \text {. }\end{array}$ & $240 \mathrm{~nm}$ & $\begin{array}{l}\text { Standard and pharmaceutical } \\
\text { form }\end{array}$ & [25] \\
\hline HPLC-UV & $\begin{array}{l}\text { Column shimpack GLC-ODS ( } 150 \mathrm{~mm} \times 6 \mathrm{~mm} ; 5 \mu \mathrm{m}) \text {; at ambient } \\
\text { temperature. Mobile phase: acetonitrile and } 0.1 \mathrm{M} \text { ammonium } \\
\text { acetate solution }(10: 90 \mathrm{v} / \mathrm{v}), \mathrm{pH} 7.5 \text { using ammonium solution; for } \\
\text { alkaline degradation was used phosphate buffer }(\mathrm{pH} \text { 10) with } \\
\text { heating; flow rate } 1.5 \mathrm{~mL} / \mathrm{min} \text {; injection volume } 50 \mu \mathrm{L} \text {. }\end{array}$ & $270 \mathrm{~nm}$ & $\begin{array}{l}\text { Powder for injectable solutions } \\
\text { and their degradation } \\
\text { products }\end{array}$ & [43] \\
\hline HPLC-UV & $\begin{array}{l}\text { Analytical column Spherisorb ODS C18 }(250 \mathrm{~mm} \times 4.6 \mathrm{~mm} ; 10 \mu \mathrm{m}) \text {. } \\
\text { Mobile phase: monopotassiumphosphate buffer pH } 2.5 \text { and } \\
\text { methanol }(70: 30 \mathrm{v} / \mathrm{v}) \text {; flow rate } 1 \mathrm{~mL} / \mathrm{min} \text {; injection volume } 10 \mu \mathrm{L} \text {. }\end{array}$ & $254 \mathrm{~nm}$ & Standard & [40] \\
\hline HPLC-UV & $\begin{array}{l}\text { Column sphere-Image } 80-5 \text { ODS } 2(250 \mathrm{~mm}) \text {, at } 30^{\circ} \mathrm{C} \text {. Mobile phase: } \\
\text { acetonitrile and aqueous } 0.1 \mathrm{M} \text { citrate buffer with } 5 \mathrm{mM} \\
\text { ammonium perchlorate and } 2 \mathrm{mM} \text { tetrabutylammoniumhydrogen } \\
\text { sulfate }(11: 89 \mathrm{v} / \mathrm{v}) \text {. }\end{array}$ & $265 \mathrm{~nm}$ & Plasma and bone & [27] \\
\hline HPLC-UV & $\begin{array}{l}\text { Column waters } \times \text { bridge } \mathrm{C} 18 \mathrm{BEH}(50 \mathrm{~mm} \times 3 \mathrm{~mm} ; 2.5 \mu \mathrm{m}) \text {, at } 40^{\circ} \mathrm{C} \text {. } \\
\text { Mobile phase: } 100 \mathrm{mM} \text { o-phosphoricacid and/or sodium } \\
\text { dihydrogen-phosphate with } \mathrm{NaOH} \text { pH } 2.55 \text { and acetonitrile }(100: 12 \\
\mathrm{v} / \mathrm{v}) \text {. }\end{array}$ & $260 \mathrm{~nm}$ & Plasma & [28] \\
\hline HPLC-UV & $\begin{array}{l}\text { Column C18 }(250 \mathrm{~mm} \times 4.6 \mathrm{~mm} ; 5 \mu \mathrm{m}) \text {; at ambient temperature. } \\
\text { Mobile phase: acetonitrile, methanol and triethylamine (TEA) buffer } \\
(\mathrm{pH} 7)(1: 1: 2 \mathrm{v} / \mathrm{v}) \text {, flow rate } 0.6 \mathrm{~mL} / \mathrm{min} \text {, injection volume } 20 \mu \mathrm{L} \text {. }\end{array}$ & $240 \mathrm{~nm}$ & Biological sample & [30] \\
\hline HPLC-UV & $\begin{array}{l}\text { Column Kromasil C18 }(250 \mathrm{~mm} \times 4.6 \mathrm{~mm} ; 5 \mu \mathrm{m}) \text {. Mobile phase: } \\
1.5 \mathrm{mM} \text { potassium dihydrogen phosphate (adjust the } \mathrm{pH} \text { to } 4.5 \\
\text { with phosphoric acid) with } 0.0125 \% \text { triethylamine }- \text { methanol } \\
(70: 30, \mathrm{v} / \mathrm{v}) \text {; flow rate } 1 \mathrm{~mL} / \mathrm{min} \text {; injection volume } 20 \mu \mathrm{L} \text {. }\end{array}$ & $247 \mathrm{~nm}$ & Human urine & [31] \\
\hline HPLC-UV & $\begin{array}{l}\text { Column C18 }(30 \mathrm{~mm} \times 4.6 \mathrm{~mm} ; 2.5 \mu \mathrm{m}) \text { at ambient temperature. } \\
\text { Mobile phase: acetonitrile and } 50 \mathrm{mM} \text { phosphate buffer at } \mathrm{pH} 2.4 \\
(8: 92 \mathrm{v} / \mathrm{v}) \text {; flow rate } 1 \mathrm{~mL} / \mathrm{min} \text {; injection volume } 25 \mu \mathrm{L} \text {. }\end{array}$ & $260 \mathrm{~nm}$ & Human plasma & [32] \\
\hline HPLC-UV & $\begin{array}{l}\text { Column Atlantis T3 (150 mm } \times 4.6 \mathrm{~mm} ; 5 \mu \mathrm{m} \text { ) Mobile phase: } 10 \mathrm{mM} \\
\text { phosphoric acid solution, adjusted to } \mathrm{pH} 2 \text { with hydrochloric acid, } \\
\text { and acetonitrile (gradient elution). }\end{array}$ & $230 \mathrm{~nm}$ & Human plasma & [33] \\
\hline HPLC-UV & $\begin{array}{l}\text { Column C18 Atlantis }(150 \mathrm{~mm} \times 4.6 \mathrm{~mm} ; 3 \mu \mathrm{m}) \text {, at } 35^{\circ} \mathrm{C} \text {. Mobile } \\
\text { phase: } 50 \mathrm{mM} \text { monopotassium phosphate and acetonitrile } \\
\text { (gradient elution); flow rate } 1 \mathrm{~mL} / \mathrm{min} \text {. }\end{array}$ & $274 \mathrm{~nm}$ & Bone & [34] \\
\hline HPLC-UV & $\begin{array}{l}\text { Column C18 }(30 \mathrm{~mm} \times 4.6 \mathrm{~mm} ; 2.5 \mu \mathrm{m}) \text {, at ambient temperature. } \\
\text { Mobile phase: acetonitrile and } 50 \mathrm{mM} \text { phosphate buffer at } \mathrm{pH} 2.4 \\
(8: 92 \mathrm{v} / \mathrm{v}) \text {. }\end{array}$ & $260 \mathrm{~nm}$ & Human plasma & [37] \\
\hline HPLC-UV & $\begin{array}{l}\text { Column inertsil ODS-3 }(250 \mathrm{~mm} \times 4.6 \mathrm{~mm} ; 5 \mu \mathrm{m}) \text {; at ambient } \\
\text { temperature. Mobile phase: methanol and } 10 \mathrm{mM} \text { dipotassium } \\
\text { phosphate buffer at } \mathrm{pH} 6.7(21: 79, \mathrm{v} / \mathrm{v}) \text {; flow rate } 1.1 \mathrm{~mL} / \mathrm{min} \text {; } \\
\text { injection volume } 20 \mu \mathrm{L} \text {. }\end{array}$ & $270 \mathrm{~nm}$ & $\begin{array}{l}\text { Rat plasma and intervertebral } \\
\text { disc }\end{array}$ & [38] \\
\hline HPLC-UV & $\begin{array}{l}\text { Column } \times \text { Terra } \mathrm{C} 18(250 \mathrm{~mm} \times 4.6 \mathrm{~mm} ; 5 \mu \mathrm{m}) \text { at } 32^{\circ} \mathrm{C} \text {. Mobile } \\
\text { phase: } 40 \mathrm{mM} \text { phosphate buffer }(\mathrm{pH} 3.2) \text { and methanol (gradient } \\
\text { mode); flow rate } 0.85 \mathrm{~mL} / \mathrm{min} \text {; injection volume } 20 \mu \mathrm{L} \text {. }\end{array}$ & ND & Plasma and amniotic fluid & [39] \\
\hline HPLC-UV & $\begin{array}{l}\text { Column Nova-Pak C18 ( } 100 \mathrm{~mm} \times 8 \mathrm{~mm} ; 4 \mu \mathrm{m}) \text {. Mobile phase: } \\
10 \mathrm{Mm} \text { of dibasicpotassium phosphate and } 10 \mathrm{mM} \text { cetyltrimethyl } \\
\text { ammonium bromide (pH 6.5) with acetonitrile (73:27 v:v). }\end{array}$ & $274 \mathrm{~nm}$ & Plasma & [41] \\
\hline HPLC-UV & $\begin{array}{l}\text { Column C18 Hypersyl ( } 200 \mathrm{~mm} \times 2.1 \mathrm{~mm} ; 5 \mu \mathrm{m}) \text {; at ambient } \\
\text { temperature. Mobile phase: for the analysis of the drug in the } \\
\text { aqueous system, methanol-acetonitrile-phosphate buffer, } \mathrm{pH} 7.4 \\
(20: 20: 60, \mathrm{v} / \mathrm{v} / \mathrm{v}) \text {; for the plasma and cerebrospinal fluid, }(30: 40: 30 \text {, } \\
\mathrm{v} / \mathrm{v} / \mathrm{v}) ; \text { flow rate } 0.5 \mathrm{~mL} / \mathrm{min} \text {. }\end{array}$ & $270 \mathrm{~nm}$ & $\begin{array}{l}\text { Aqueous and rabbit biological } \\
\text { samples }\end{array}$ & [42] \\
\hline HPLC-UV & $\begin{array}{l}\text { Column supelcosil LC-18 }(150 \mathrm{~mm} \times 4.6 \mathrm{~mm} ; 3 \mu \mathrm{m}) \text {; at ambient } \\
\text { temperature. Two analytical mobile phases were used: mobile } \\
\text { phase I: methanol -acetonitrile }-0.01 \mathrm{M} \text { phosphate buffer }(\mathrm{pH} 7.0) \\
\text { (20:15:65) and } 5 \mathrm{mM} \text { tetrabutyl ammonium hydrogensulfate; } \\
\text { mobile phase II: acetonitrile was omitted and } 30 \% \text { of methanol was } \\
\text { used; flow rate } 1 \mathrm{~mL} / \mathrm{min} \text {; injection volume } 20 \mu \mathrm{L} \text {. }\end{array}$ & $267 \mathrm{~nm}$ & Serum concentrations & [44] \\
\hline
\end{tabular}


Table 1. (Continued)

\begin{tabular}{|c|c|c|c|c|}
\hline Method & Conditions & Detection system & Matrices & Reference \\
\hline HPLC-UV & $\begin{array}{l}\text { Column p-Bondapak C18. Mobile phase: } 56 \% 0.07 \mathrm{M} \\
\text { sodiumacetateand } 44 \% \text { acetonitrile, } \mathrm{pH} \\
\text { ofthesolutionwasadjustedto } 5.7 \text { with acetic cid; flow rate } 1 \mathrm{~mL} / \mathrm{min} \text {. }\end{array}$ & $270 \mathrm{~nm}$ & Plasma and urine & [45] \\
\hline HPLC-UV & $\begin{array}{l}\text { Column hypersil ODS }(250 \mathrm{~mm} \times 4.6 \mathrm{~mm} ; 5 \mu \mathrm{m}) \text {, at } 50^{\circ} \mathrm{C} \text {. Mobile } \\
\text { phase: acetonitrile: water: } \mathrm{pH} 7: \mathrm{pH} 5(39-55-5.5-0.5) \text {; flow rate } \\
1.5 \mathrm{~mL} / \mathrm{min} \text {; injection volume } 20 \mu \mathrm{L} \text {. }\end{array}$ & $254 \mathrm{~nm}$ & $\begin{array}{l}\text { Residues on stainless steel } \\
\text { surface of pharmaceutical } \\
\text { manufacturing equipments }\end{array}$ & [35] \\
\hline HPLC-MS & $\begin{array}{l}\text { Column cosmosil packed HILIC }(150 \mathrm{~mm} \times 2.0 \mathrm{~mm}) \text { at } 30^{\circ} \mathrm{C} \text {. Mobile } \\
\text { phase: acetonitrile and } 10 \mathrm{mMol} / \mathrm{L} \text { ammonium acetate }(7: 3) \text {, flow } \\
\text { rate } 0.5 \mathrm{~mL} / \mathrm{min} \text {; injection volume } 10 \mu \mathrm{L} \text {. }\end{array}$ & $\begin{array}{l}\text { Electrospray ionization (ESI) } \\
\text { operated in negative mode }\end{array}$ & Powder for injectable solutions & [46] \\
\hline HPLC-MS & $\begin{array}{l}\text { Column 1: DevelosilPhA }(150 \mathrm{~mm} \times 0.5 \mathrm{~mm} ; 5 \mu \mathrm{m}) 2 \text { : Develosil PhA } \\
(30 \mathrm{~mm} \times 0.5 \mathrm{~mm} ; 10 \mu \mathrm{m}) . \text { Mobile phase: } 1: \text { deionized water, } \\
\text { methanol, acetic acid, glycerol pH } 3.0(59: 40: 0.5: 0.5 \mathrm{v} / \mathrm{v}) 2: \\
\text { deionized water, methanol, acetic acid, diethanolamine } \mathrm{pH} 3.0 \\
(57: 40: 2.5: 0.5 \mathrm{v} / \mathrm{v})\end{array}$ & $\begin{array}{l}\text { Operated in positive and } \\
\text { negative mode }\end{array}$ & Human serum & [47] \\
\hline UPLC-UV & $\begin{array}{l}\text { Column Acquity UPLC BEH C18 (100 mm } \times 2.1 \mathrm{~mm} ; 1.7 \mu \mathrm{m}) \text {. Mobile } \\
\text { phase: Solution A containing potassium dihydrogenphosphate } \\
\text { buffer (pH adjusted to } 6.5 \pm 0.2 \text { with } 0 \text {-phosphoric acid), citric acid } \\
\text { buffer (pH adjusted to } 5.0 \pm 0.2 \text { with } \mathrm{NaOH} \text { solution) and } \\
\text { acetonitrile and solution B containing tetradecyl ammonium } \\
\text { bromide, tetraheptyl ammonium bromide and acetonitrile. } \\
\text { Solution A: Solution B } 65: 35(\mathrm{v} / \mathrm{v}) \text {; flow rate } 0.3 \mathrm{~mL} / \mathrm{min} \text {. }\end{array}$ & $230 \mathrm{~nm}$ & Powder for injectable solutions & [50] \\
\hline UPLC-UV & $\begin{array}{l}\text { Column purospher star C18 (100 mm } \times 2.1 \mathrm{~mm} ; 2 \mu \mathrm{m}) \text {. Mobile phase: } \\
0.05 \mathrm{M} \text { sodium dihydrogen } 0 \text {-phosphate dihydrate: acetonitrile } \\
(86: 14 \mathrm{v} / \mathrm{v}) \text { adjusted to } \mathrm{pH} 4.5 \text { with } 0.1 \mathrm{M} \text { o-phosphoric or } 0.1 \mathrm{M} \\
\text { sodium hydroxide; flow rate } 0.4 \mathrm{~mL} / \mathrm{min} \text {. }\end{array}$ & $254 \mathrm{~nm}$ & $\begin{array}{l}\text { Powder for injectable } \\
\text { solutions }\end{array}$ & [51] \\
\hline UPLC-UV & $\begin{array}{l}\text { Column acquity UPLC BEH C18 }(50 \mathrm{~mm} \times 2.1 \mathrm{~mm}, 1.7 \mu \mathrm{m}) \text {. Mobile } \\
\text { phase: acetonitrile and } 10 \mathrm{mM} \text { oxalic acid (gradient elution); } \\
\text { flow rate of } 0.35 \mathrm{~mL} / \mathrm{min} \text {; injection volume } 5 \mu \mathrm{L} \text {. }\end{array}$ & $\begin{array}{l}380 \mathrm{~nm}(0-0.58 \mathrm{~min}), 268 \mathrm{~nm} \\
(0.58-1.01 \mathrm{~min}), 229 \mathrm{~nm} \\
(1.01-1.12 \mathrm{~min}) \text { and } 268 \mathrm{~nm} \\
(1.12-6.00 \mathrm{~min})\end{array}$ & Residues in milk & [52] \\
\hline UPLC-MS/MS & $\begin{array}{l}\text { Column AcquityUplcBehC18 }(50 \mathrm{~mm} \times 2.1 \mathrm{~mm} ; 1.7 \mu \mathrm{m}) \text { at } 35^{\circ} \mathrm{C} \text {. } \\
\text { Mobile phase: } 0.1 \% \text { formic acid in ultra-pure water and } 0.1 \% \\
\text { formic acid in acetonitrile (gradient mode); flow rate } 0.4 \mathrm{~mL} / \mathrm{min} \text {. }\end{array}$ & $\begin{array}{l}\text { Positive electrospray ionization } \\
\text { (ESI) using multiple reaction } \\
\text { monitoring (MRM) }\end{array}$ & Waste water & [48] \\
\hline UPLC-MS/MS & $\begin{array}{l}\text { ColumnC }{ }_{18} \text { Wwaters acquity T3 }(50 \mathrm{~mm} \times 2.1 \mathrm{~mm} ; 1.7 \mu \mathrm{m}) \text {. Mobile } \\
\text { phase: } 0.1 \% \text { formic acid in water and } 0.1 \% \text { formic acid in } \\
\text { acetonitrile (gradient mode); flow rate } 0.4 \mathrm{~mL} / \mathrm{min} \text {; injection } \\
\text { volume } 5 \mu \mathrm{L} \text {. }\end{array}$ & $\begin{array}{l}\text { Positive electrospray ionization } \\
\text { (ESI) using multiple reaction } \\
\text { monitoring (MRM) }\end{array}$ & Blood & [49] \\
\hline
\end{tabular}

HPLC-UV: High-performance liquid chromatography with detection by ultraviolet; HPLC-MS: High-performance liquid chromatography coupled with mass spectrometry detection; UPLC-MS/MS: Ultra-performance liquid chromatography coupled with sequential mass spectrometry; HPLC-MS/MS: High-performance liquid chromatography coupled with sequential mass spectrometry and UPLC-UV: Ultra-performance liquid chromatography with detection by ultraviolet; ND: not declared.

monitoring of the substance. In the analyzed literature, there is a predominance of determination by high-performance liquid chromatography (HPLC), but there are also determinations using ultra-performance liquid chromatography (UPLC), absorption spectroscopy ultravioleta (UV), infrared spectroscopy (IV), spectrofluorimetry and microbiological methods.

Table 2. Spectrometric analytical methods described in the literature for the determination of ceftriaxone sodium.

\begin{tabular}{|c|c|c|c|c|}
\hline Method & Conditions & Detection system & Matrices & Reference \\
\hline $\begin{array}{l}\text { Absorption } \\
\text { spectroscopy VIS }\end{array}$ & $\begin{array}{l}\text { Dilution in water until the concentration of } 2-8 \mathrm{mg} \mathrm{mL}^{-1} \text { with } 4 \text {-chloro-7- } \\
\text { nitrobenzo-2-oxa-1,3-diazole. }\end{array}$ & $390 \mathrm{~nm}$ & Powder for injectable solutions & [53] \\
\hline $\begin{array}{l}\text { Absorption } \\
\text { spectroscopy VIS }\end{array}$ & $\begin{array}{l}\text { Dilution of } 250 \mathrm{mg} \text { of sample in water, after filtration was diluted with } \\
\text { distilled water and made up to } 100 \mathrm{~mL} \text {. Using variamine blue for } \\
\text { determination. }\end{array}$ & $556 \mathrm{~nm}$ & Commercial productcs & [54] \\
\hline $\begin{array}{l}\text { Absorption } \\
\text { spectroscopy VIS }\end{array}$ & $\begin{array}{l}\text { Dilutions of the drug with methanol and acetonitrile, using chloranilic acid } \\
\text { for determination. }\end{array}$ & $520 \mathrm{~nm}$ & Pharmaceutical formulation & [55] \\
\hline $\begin{array}{l}\text { Absorption } \\
\text { spectroscopy VIS }\end{array}$ & $\begin{array}{l}\text { Metallic chromium }(\mathrm{VI}) \text { reagent isoxidized directly by potassium dichromate } \\
\text { in the presence of the drug in acid medium forming a ternary complex. }\end{array}$ & $520 \mathrm{~nm}$ & Commercial formulation & [6] \\
\hline $\begin{array}{l}\text { Absorption } \\
\text { spectroscopy UV }\end{array}$ & $\begin{array}{l}\text { Volumes of } 100-300 \mu \mathrm{L} \text { of ceftriaxona solutions }(1 \mathrm{mg} / \mathrm{mL}) \text { were mixed with } \\
50 \mu \mathrm{L} \text { of the alkali-induced degradation product }(1 \mathrm{mg} / \mathrm{mL}) \text { and diluted } \\
\text { to } 10 \mathrm{~mL} \text { with acetonitrile-water }(10: 90 \mathrm{v} / \mathrm{v}) \text {. }\end{array}$ & $265-320 \mathrm{~nm}$ & $\begin{array}{l}\text { Powder for injectable solutions } \\
\text { and their degradation products }\end{array}$ & [43] \\
\hline $\begin{array}{l}\text { Absorption } \\
\text { spectroscopy UV }\end{array}$ & $\begin{array}{l}\text { A portion of powder equivalent to } 100 \mu \mathrm{g} \text { of the drug was weighed and } \\
\text { transferred into } 100 \mathrm{~mL} \text { volumetric flask, the drug was dissolved by } \\
\text { adding } 70 \mathrm{~mL} \text { of distilled water and sonicated for } 15 \mathrm{~min} \text {. The volume was } \\
\text { completed with distilled water and filtered. }\end{array}$ & $241 \mathrm{~nm}$ & Powder for injection dosage forms & [56] \\
\hline Spectrofluorimetry & Dilution with water until $20 \mu \mathrm{g} / \mathrm{mL}$ reacting with $o$-phthalaldehyde. & $\begin{array}{l}386 \text { and } \\
324 \mathrm{~nm}\end{array}$ & $\begin{array}{l}\text { Pharmaceutical formulations and } \\
\text { plasma }\end{array}$ & [57] \\
\hline Infrared & $\begin{array}{l}\text { It was linear over the concentration range of } 0.4-1.2 \mathrm{mg} \text { with correlation } \\
\text { coefficient of } 0.9983 \text { and limits of detection and quantification of } 0.016 \\
\text { and } 0.049 \mathrm{mg}\end{array}$ & $\begin{array}{l}1800 \text { and } \\
1700 \mathrm{~cm}^{-1}\end{array}$ & Powder for injectable solutions & [59] \\
\hline
\end{tabular}


Table 3. Microbiological analytical methods described in the literature for the determination of ceftriaxone sodium.

\begin{tabular}{|c|c|c|c|c|}
\hline Method & Conditions & $\begin{array}{l}\text { Incubation } \\
\text { time (hour) }\end{array}$ & Matrices & Reference \\
\hline $\begin{array}{l}\text { Microbiological- } \\
\text { diffusion in agar }\end{array}$ & Plate cylinder method, $3 \times 3$ using Staphylococcus aureus ATCC 6538P. & 18 & Standard & [7] \\
\hline $\begin{array}{l}\text { Microbiological- } \\
\text { diffusion in agar }\end{array}$ & $\begin{array}{l}\text { Samples containing concentrations of } 4.0 \mu \mathrm{g} / \mathrm{mL} \text {, using phosphate buffer } \mathrm{pH} 6.0 \text { as diluent; } \\
\text { plates containing } 21 \mathrm{~mL} \text { of medium for antibiotic assay as base layer and } 4 \mathrm{~mL} \text { of the same } \\
\text { medium inoculated with Bacillus subtilis (ATCC 6633) in a proportion of } 0.5 \% \text {; and } \\
\text { incubation at } 37 \pm 1^{\circ} \mathrm{C} \text { for } 18 \text { hours. }\end{array}$ & 18 & Commercial productcs & [9] \\
\hline $\begin{array}{l}\text { Microbiological- } \\
\text { diffusion in agar }\end{array}$ & $\begin{array}{l}\text { Six plates were used for each assay with Bacillus subtilis ATCC } 9371 \text { IAL } 1027 \text { as test } \\
\text { microorganism. The plates were incubated at } 35^{\circ} \mathrm{C} \text { aerobically for } 18 \text { hours. The diameter } \\
\text { of the growth inhibition zone }(\mathrm{mm}) \text { was carefully measured with a digital caliper. }\end{array}$ & 18 & $\begin{array}{l}\text { Powder for Injectable } \\
\text { Solution }\end{array}$ & [58] \\
\hline
\end{tabular}

Tables 1-3 present the chromatographic, spectrometric and microbiological analytical methods, respectively, described in the literature for the determination of ceftriaxone sodium in pharmaceutical formulations, standards and various biological matrices.

Figure 3 shows graphically the percentage of different methods used for the analysis of ceftriaxone sodium, the HPLC technique was the most used in the found methods, being a fast, precise and specific technique but uses organic solvents (acetonitrile, methanol, for example), being toxic waste generators. Many methods use buffer solutions that are non-toxic to the environment and the operator, but can shorten the life of the equipment and accessories, for example, the chromatographic columns, impacting the cost of the analysis. ${ }^{[60-61]}$

It is necessary to develop methods that use solvents with low toxicity (ethanol and water, for example), as well as to use them in low concentrations. The commitment to using reduced samples through miniaturization, decreasing process steps and pretreatment of samples, are alternatives that directly influence the amount of reagents used, time of analysis, number of materials required and cost involved. On the other hand, work on the recovery of these toxic solvents should be done, as these materials cannot be disposed of directly into the environment. ${ }^{\text {[62-65] }}$

The equipment used should also be considered important, the proposal is to use those that require less solvent, which have less analysis time, generating lower energy consumption and as a consequence lower expenses for the company and lower final product prices. ${ }^{[66-70]}$

\section{Analytical Methods}

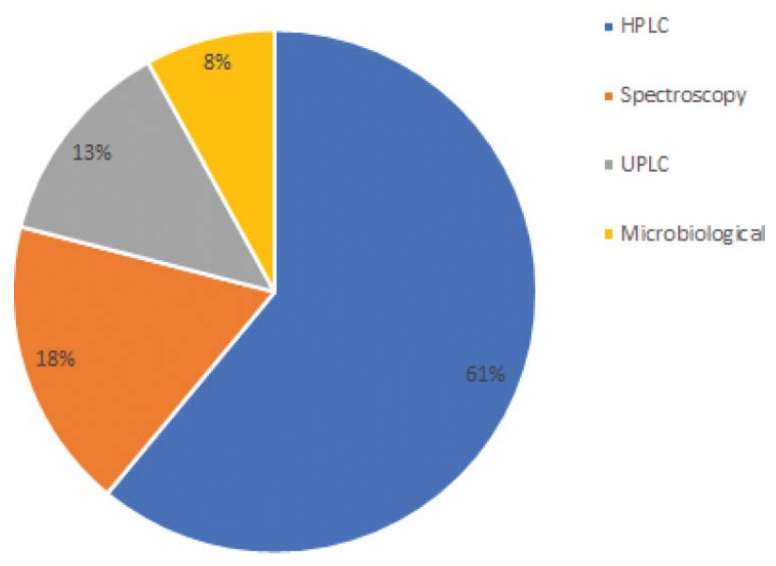

Figure 3. Percentage of various analytical methods used in the ceftriaxone sodium analysis.
The questions tend to focus on the relationship between the new validated methods and their practical uses by chemical and pharmaceutical industries. In discussing the relevant issue, we have to consider how methods can be adopted to routine analysis. This is clearly not an exhaustive list, but it will be very useful for researchers to revise the key concepts and demonstrate the ability of these innovative techniques. The development of these methods should be encouraged more and more, due to their advantages and economic, environmental and social benefits. In this way, universities become reference research centers in the area, helping to achieve this goal. ${ }^{[3,4,15,71-74]}$

\section{Conclusion}

Ceftriaxone sodium is a third-generation antibiotic belonging to a class of cephalosporins, it has a bactericidal action and it shows greater activity against Gram-negative bacteria.

The use of this drug contributes to the development of studies that need to carry out their analytical and bioanalytical quantification. The drug has characteristics, properties and analytical methods well defined. However, it is necessary to encourage and raise awareness for the need to develop and validate innovative analytical methods using green chemistry.

\section{Conflict of interest}

The authors declare no conflicts of interest.

\section{Acknowledgments}

The authors acknowledge to CAPES and CNPq (Brasília, Brazil).

\section{Funding}

Conselho Nacional de Desenvolvimento Científico e Tecnológico (304824/ 2013-5).

\section{ORCID}

Mariana Teixeira da Trindade (ID) http://orcid.org/0000-0003-0745-0277 Hérida Regina Nunes Salgado (iD http://orcid.org/0000-0002-0385-340X

\section{References}

[1] Guimaraes, D. O.; Momesso, L. S.; Pupo, M. T. Antibiotics Therapeutic Importance and Prospects for the Discovery and Development of New Agents. New Chem. 2010, 33, 667-679. 
[2] Fernandes, R.; Amador, P.; Prudêncio, C. $\beta$-Lactams: Chemical Structure, Mode of Action and Mechanisms of Resistance. Rev. Med. Microbiol. 2013, 24, 7-17.

[3] Consortti, L. P.; Salgado, H. R. N. A Critical Review of Analytical Methods for Quantification of Cefotaxime. Crit. Rev. Anal. Chem. 2017, 47, 359-371.

[4] Marco, B. A.; Salgado, H. R. N. Characteristics, Properties and Analytical Methods of Cefadroxil: A Review. Crit. Rev. Anal. Chem. 2017, 47, 2, 93-98.

[5] Vieira, D. C. M.; Ricarte, P. C.; Salgado, H. R. N. Development and Validation of the Quantitative Analysis of Cefuroxime Sodium in Powder for Injection by Infrared Spectroscopy. Adv. Anal. Chem. 2012, 6, 80-87.

[6] Amin, A. S.; Ragab, G. H. Spectrophotometric Determination of Certain Cephalosporins in Pure Form and in Pharmaceutical Formulations. Spectrochim. Acta Part A. 2004, 60, 2831-2835.

[7] Manfio, M. L.; Agarrayua, D. A.; Machado, J. C.; Schmidt, C. A. A Fully Validated Microbiological Assay to Evaluate the Potency of Ceftriaxone Sodium. Braz. J. Pharm. Sci. 2013, 49, 4.

[8] Ali, S. M.; Elbashir, A. A.; Aboul-Enein, H. Y. Spectroscopic Methods for Analysis of Cephalosporins in Pharmaceutical Formulations. World J. Anal. Chem. 2015, 3, 21-32.

[9] Lourenço, F. R.; Traple, M. A.; Okamoto, R. T.; Pinto, T. J. A. Development and Validation of Microbiological Assay for Ceftriaxone and its Application in Photo-Stability Study. Curr. Pharma. Anal. 2013, 9, 77-81.

[10] Brayfield, A. Martindale: The Complete Drug Reference 38th ed; Pharmaceutical Press: London, United Kingdom, 2014.

[11] Norival, C. BPR.Guide of Medicines; Escala: São Paulo, Brazil, 2014.

[12] Melo, J. M. S. DEF. Pharmaceutical Speciality Dictionary; Epuc: São Paulo, Brazil, 2015.

[13] ROCEFIN ${ }^{\mathrm{TM}}$ http://www.anvisa.gov.br/datavisa/fila_bula/frmVisuali zarBula.asp?pNuTransacao $=4844762015 \&$ pIdAnexo $=2658383$ (accessed July 11, 2017).

[14] La Roca, M. F.; Sobrinho, J. L. S.; Nunes, L. C. C; Neto, P. J. R. Development and Validation of Analytical Method: Important Step in the Production of Medicines. Brazilian J. Pharm. 2007, 88, 177-180.

[15] Silva, L. M.; Salgado, H. R. N. Tigecycline: A Review of Properties, Applications, and Analytical Methods. Ther. Drug Monit. 2010, 32, 282-288.

[16] Owens, H. M.; Dash, A. Ceftriaxone Sodium: Comprehensive Profile; Profiles of Drug Substances Ecipients and Related Methodology: Omaha, USA, 2003.

[17] BP. British Pharmacopoeia; The Stationary Office: London, 2013.

[18] EP. European Pharmacopoeia, 8 ed.; Council of Europe: Strasbourg, 2013.

[19] JP. Japanese Pharmacopoeia, 16th ed.; Society of Japanese Pharmacopoeia: Tokyo, 2011.

[20] Portuguese Pharmacopoeia, 7 ed; Infarmed: Lisboa, 2005.

[21] SP. Spanish Pharmacopoeia, 3 ed.; Madrid, Ministerio de Sanidad y Consumo, Agencia Española de Medicamentos y Productos Sanitarios, 2007.

[22] USP 39. The United States Pharmacopeia, 39th ed; Rockville: United States Convention, 2016.

[23] Katzung, B. G. Basic Pharmacology \& Clinical, 9th ed; Guanabara Koogan S.A.: Rio de Janeiro, Brazil, 2005.

[24] Tavares, W. Manual of Antibiotics and Chemotherapeutic Anti-Infectives, 2nd ed.; Atheneu: São Paulo, 1996.

[25] Hiremath, B.; Mruthyunjayaswamy, B. H. M. Development and Validation of a High Performance Liquid Chromatographic Determination of Ceftriaxone Sodium and its Application to Drug Quality Control. Anal. Letters. 2009, 42, 2180-2190.

[26] Drug Bank. Drug \& Drug Target Database. https://www.drugbank. ca/drugs/DB01212 (accessed July 11, 2017).

[27] Gergs, U.; Clauss, T.; Ihlefeld, D.; Weiss, M.; Pönicke, K.; Hofmann, G. O.; Neumann, J. Pharmacokinetics of Ceftriaxone in Plasma and Bone of Patients Undergoing Hip or Knee Surgery. J. Pharm. Pharmacol. 2014, 66, 1552-1558.

[28] Kratzer, A.; Liebchen, U.; Schleibinger, M.; Kees, M. G.; Kees, F. Determination of Free Vancomycin, Ceftriaxone, Cefazolin
Andertapenem in Plasma by Ultrafiltration: Impact of Experimental Conditions. J. Chromatogr. B. 2014, 961, 97-102.

[29] El-Adl, S. M.; El-Shanawany, A. A.; Abdel-Aziz, L. M.; Hassan, A. F. HPLC Determination of Three Cephalosporins (Cefepime, Cefotriaxone and Cefotaxime) in their Bulk and Dosage Forms. Asian J. Pharm. Anal. 2014, 4, 91-97.

[30] Shah, J.; Jan, M. R.; Shah, S.; Khan, M. N. Development and Validation of HPLC Method for Simultaneous Determination of Ceftriaxone and Cefaclor in Commercial Formulations and Biological Samples. J. Mexican Chem. Soc. 2013, 57, 314-320.

[31] Sun, H.; Wang, H.; Ge, X. Simultaneous Determination of the Combined Drugs of Ceftriaxone Sodium, Metronidazole, and Levofloxacin in Human Urine by High-Performance Liquid Chromatography. J Clin. Lab. Anal. 2012, 26, 486-492.

[32] Briscoe, S. E.; McWhinney, B. C.; Lipman, J.; Roberts, J. A.; Ungerer, J. P. J. A Method for Determining the Free (Unbound) Concentration of Ten Beta-Lactam Antibiotics in Human Plasma Using High Performance Liquid Chromatography with Ultraviolet Detection. J. Chromatogr. B. 2012, 907, 178-184.

[33] Verdier, M. C.; Tribut, O.; Tattevin, P.; Tulzo, Y. L.; Michelet, C.; Ferrer, D. B. Simultaneous Determination of $12 \beta$-Lactam Antibiotics in Human Plasma by High Performance Liquid Chromatography with UV Detection: Application to Therapeutic Drug Monitoring. Antimicrob. Agents Chemother. 2011, 10, 4873-4879.

[34] Garazzino, S.; Aprato, A.; Baietto, L.; Avolio, A. D.; Maiello, A.; Rosa, F. G. D.; Aloj, D.; Siccardi, M.; Biasibetti, A.; Massè, A.; Perri, G. D. Ceftriaxone Bone Penetration in Patients with Septic Non-Union of the Tíbia. Int. J. Infect. Dis. 2011, 15, 415-421.

[35] Akl, M. A.; Ahmed, M. A.; Ahmed, R. Validation of an HPLC-UV Method for the Determination of Ceftriaxone Sodium Residues on Stainless Steel Surface of Pharmaceutical Manufacturing Equipments. J. Pharm. Biomed. Anal. 2011, 55, 247-252.

[36] Kale, R. S.; Jain, H. K.; Prashant, D. G.; Mhaske, G. S.; Puri, M. V.; Raut, M. D.; Patil, H. S. An RPHPLC Method for Simultaneous Estimation of Ceftriaxone Sodium and Sulbactam Sodium in Parenteral Dosage Form. Int. J. Pharm. Pharm. Sci. 2011, 3, 406-409.

[37] McWhinney, B.; Wallis, S. C.; Hillister, T.; Roberts, J. A.; Lipman, J.; Ungerer, J. P. J. Analysis of 12 Beta-Lactam Antibiotics in Human Plasma by HPLC with Ultraviolet Detection. J. Chromatogr. B. 2010, 878, 2039-2043.

[38] Lin, C. C.; Wu, Y. T.; Yen, J. C.; Chiang, C. J.; Tsuang, Y. H.; Tsai, T. H. In Vitro and In Vivo Methods to Measure the Ceftriaxone Distribution into the Rat Tail Intervertebral Disc. Anal. Sci. 2010, 26, 979-982.

[39] Nemutlua, E.; Kir, S.; Katlanb, D.; Beksac, M. S. Simultaneous Multiresponse Optimization of an HPLC Method to Separate Seven Cephalosporins in Plasma and Amniotic Fluid: Application to Validation and Quantification of Cefepime, Cefixime and Cefoperazone. Talanta 2009, 80, 117-126.

[40] Doadrio, A. L.; Mayorga, A.; Madrigal P.; Orenga, R. Determinación de las constantes de la relacción de hidrólisis de ceftriaxona por cromatografia de líquidos. An de la Real Academia de Farm. 2001, 67, 27-36.

[41] Al-Rawithi, S.; Hussein, R.; Raines, D. A.; AlShowaier, I.; Kurdi, W. Sensitive Assay for the Determination of Cefazolin or Ceftriaxone in Plasma Utilizing LC. J. Pharm. Biomed. Anal. 2000, 22, 281-286.

[42] Owens, H. M.; Destache, C. J.; Dash, A. K. Simple Liquid Chromatographic Method for the Analysis of the Blood Brain Barrier Permeability Characteristics of Ceftriaxone in an Experimental Rabbit Meningitis Model. J. Chromatogr. B. 1999, 728, 97-105.

[43] Abdel-Hamid, M. E. FSQ Spectrophotometric and HPLC Analysis of Some Cephalosporins in the Presence of their Alkali-Induced Degradation Products. Il Farmaco. 1998, 53, 132-138.

[44] Bompadre, S.; Ferrante, L.; Leone, L. On-Line Solid-Phase Extraction of Cephalosporins. J. Chromatogr. A. 1998, 812, 191-196.

[45] Paradis, D. P.; Vallée, F.; Allard, S.; Bisson, C.; Daviau, N.; Drapeau, C.; Auger, F.; Lebel, M. Comparative Study of Pharmacokinetics and Serum Bactericidal Activities of Cefpirome, Ceftazidime, Ceftriaxone, Imipenem, and Ciprofloxacin. Antimicrob. Agents Chemother. 1992 36, 2085-2092. 
[46] Tange, M.; Yoshida, M.; Nakai, Y.; Uchida, T. The Role of an Impurity in Ceftriaxone Sodium Preparation for Injection in Determining Compatibility with Calcium-Containing Solutions. Chem. Pharm. Bull. 2016, 64, 207-214.

[47] Kobayashi, K.; Sato, K.; Mizuno, Y.; Katsumata, Y. Capillary HighPerformance Liquid Chromatography-Fast Atom Bombardment Mass Spectrometry of 24 Cephem Antibiotics. J. Chrom. B. 1996, 677, 275-290.

[48] Yu, X.; Tang, X.; Zuo, J.; Zhang, M.; Chen, L.; Li, Z. Distribution and Persistence of Cephalosporins In Cephalosporin Producing Wastewater Using SPE and UPLC-MS/MS Method. Sci. Total Env. 2016, 569, 23-30.

[49] Page-Sharp, M.; Nunn, T.; Salman, S.; Moore, B. R.; Batty, K. T.; Davis, T. M. E.; Manning, L. Validation and Application of a Dried Blood Spot Ceftriaxone Assay. Antimicrob. Agents Chemother. 2016, 60, 14-23.

[50] Panchal, V. J.; Desai, H. T.; Dave, A. G. Development and Validation of Stability Indicating Method for Simultaneous Estimation of Ceftriaxone and Tazobactam Injection Using RP-UPLC Method. Int. J. Pharm. Sci. Res. 2014, 5, 2398-2405.

[51] Nirav, P. B.; Alpesh, A. C.; Sneha, J. K.; Vipul, M. D.; Hemant, D. T. Development and Validation of Stability Indicating Method for Simultaneous Estimation of Ceftriaxone and Sulbactam Injection Using RP-UPLC Method. IOSR J. Pharm. 2012, 2, 29-37.

[52] Wang, L.; Li, Y. Q. Simultaneous Determination of Ten Antibiotic Residues in Milk by UPLC. Chromatographia. 2009, 70, 253-258.

[53] Rageh, A. H.; El-Shaboury, S. R.; Saleh, G. A.; Mohamed, F. A. Spectophotometric Method for Determination of Certain Cephalosporins Using 4-Chloro-7-Nitrobenzo-2-Oxa-1, 3-Diazole (NBD-Cl). Nat. Sci. 2010, 8, 828-840.

[54] Pasha, C.; Narayana, B. A Simple Method for the Spectrophotometric Determination of Cephalosporins in Pharmaceuticals Using Variamine Blue. Eclética Química. 2008, 33, 41-46.

[55] Saleh, G. A.; Askal, H. F.; Darwish, I. A.; El-Shorbagi, A. A. Spectroscopic Analytical Study for the Charge-Transfer Complexation Of Certain Cephalosporins with Chloranilic Acid. Anal. Sci. 2003, 19, 281-287.

[56] Ethiraj, R.; Thiruvengadam, E.; Sampath, V. S.; Vahig, A.; Raj, J. Development and Validation of Stability Indicating Spectroscopic Method for Content Analysis of Ceftriaxone Sodium in Pharmaceuticals. Int. Sch. Res. Not. 2014, 2014, 278173.

[57] Shah, J.; Jan, M. R.; Shah, S.; Inayatullah. Development and Validation of a Spectrofluorimetric Method for the Quantification of Ceftriaxone in Pharmaceutical Formulations and Plasma. The J. Biol. Chem. Lumin. 2013, 28, 516-522.

[58] Aléssio, P. V.; Salgado, H. R. N. Development and Validation of a Successful Microbiological Agar Assay for Determination of Ceftriaxone Sodium in Powder for Injectable Solution. Pharmaceutics. 2012, 4, 334-342.

[59] Trindade, M. T.; Salgado, H. R. N. Development and Validation of a Modern and Stability-Indicating Method for the Quantification of Ceftriaxona Sodium in Powder for Injection by Infrared Spectroscopy. Phys. Chem. 2017, 7, 55-62. DOI:10.5923/j.pc.20170703.01.

[60] Figueiredo, A. L.; Kogawa, A. C.; Salgado, H. R. N. Development and Validation of an Ecological, New and Rapid Stability-Indicating High Performance Liquid Chromatography for Quantitative
Determination of Aztreonam in Lyophilized Powder for Injection. Drug Anal. Res. 2017, 1, 24-30. http://seer.ufrgs.br/index.php/dar/ article/view/73755/43166

[61] Marco, B. A.; Salgado, H. R. N. Development and Validation of a Green RP-HPLC Method for Quantification of Cefadroxil Capsules. World J. Pharm. Pharm. Sci. 2017, 6, 2074-2091.

[62] Figueiredo, A. L.; Salgado, H. R. N. Development and Validation of a Ultraviolet (UV) Spectrophotometric Method for Determination of Aztreonam in Pharmaceutical Products. EC Microbiol. 2017, 8, 305316.

[63] Figueiredo, A. L.; Salgado, H. R. N. Validation of a Green Analytical Method for the Quantitative Analysis of Antimicrobial Aztreonam in Lyophilized Powder for Injection by Fourier-Transform Infrared Spectroscopy (FT-IR). EC Microbiol. 2017, 8, 254-265.

[64] Aléssio, P. V.; Kogawa, A. C.; Salgado, H. R. N. Quality Of Ceftrixone Sodium in Lyophilized Powder for Injection Evaluated by Clean, Fast and Efficient Spectrophotometric Method. J. Anal. Methods in Chem. 2017, 2017, 1-4. https://doi.org/10.1155/2017/7530242.

[65] Consortti, L. P.; Salgado, H. R. N. Green Method for Quantification of Sodium Cefotaxime in Lyophilized Powder by Infrared Spectroscopy. J. Pharm. Sci. Emerging Drugs. 2017, 5, 1-6. DOI:10.4172/ 2380-9477.1000118.

[66] Kogawa, A. C.; Salgado, H. R. N. Rifaximin Stability: A Look by UV, IR, HPLC and Turbidimetry Method. J. AOAC Int. 2017. https://doi. org/10.5740/jaoacint.17-0102

[67] Kogawa, A. C.; Salgado, H. R. N. Golden Age of Green Chemistry. EC Microbiol. 2017, 12, 52-54.

[68] Kogawa, A. C.; Salgado, H. R. N. Spectrophotometry in Infrared Region: A New, Low Cost and Green Way to Analyze Tablets of Rifaximin. Curr. Pharm. Anal. 2017, 13, 1-8. DOI:10.2174/ 1573412913666161213103657

[69] Rechelo, B. S.; Fernandes, F. H. A.; Kogawa, A. C.; Pedroso, T. M.; Salgado, H. R. N. Comparative of Three Titrimetric Determinations of Cefazolin Sodium. Res. Rev.: Res. Rev. Pharm. Pharm. Sci. 2017, 6 (4), 1-8.

[70] Rechelo, B. S.; Fernandes, F. H. A.; Kogawa, A. C.; Pedroso, T. M.; Salgado, H. R. N. New Environmentally Friendly Method for Quantification of Cefazolin Sodium. Eur. Chem. Bull. 2017, 6, 238-245. DOI:10.17628/ecb.2017.6.238-245.

[71] Zimmermann, A.; Tótoli, E. G.; Fernandes, F. H. A.; Salgado, H. R. N. An Eco-Friendly and Low Cost Method for Quantification of Cefazolin Sodium in Powder for Injectable Solution Using Thin Layer Chromatography (TLC) Assisted by Digital Images. J. Planar Chromatogr. - Mod. TLC. 2017, 30, 285-290. DOI:10.1556/1006.2017.30.4.8

[72] Kogawa, A. C.; Salgado, H. R. N. Development and Validation of Infrared Spectroscopy Method for the Determination of Darunavir in Tablets. Phys. Chem. 2013, 3, 1, 1-6.

[73] Marco, B. A.; Natori, J. S. H.; Fanelli, S.; Tótoli, E. G.; Salgado, H. R. N. Characteristics, Properties and Analytical Methods of Amoxicillin: A Review with Green Approach. Crit. Rev. Anal. Chem. 2017, 47, 267-277.

[74] Pedroso, T. M.; Salgado, H. R. N. A Critical Review of Analytical Methods for Determination of Ertapenem Sodium. Crit. Rev. Anal. Chem. 2014, 46, 15-21. 